\title{
New genetic insights highlight 'old' ideas on motor dysfunction in dystonia
}

\author{
Rose E. Goodchild ${ }^{1}$, Kathrin Grundmann ${ }^{2}$, and Antonio Pisani ${ }^{3,4}$ \\ ${ }^{1}$ Vlaams Instituut voor Biotechnologie (VIB) Centre for the Biology of Disease and KU Leuven, Department of Human Genetics, \\ Campus Gasthuisberg, 3000 Leuven, Belgium \\ ${ }^{2}$ Department of Medical Genetics and Applied Genomics, University of Tuebingen, Tuebingen, Germany \\ ${ }^{3}$ Department of System Medicine, University of Rome Tor Vergata, Rome, Italy \\ ${ }^{4}$ Fondazione Santa Lucia, Istituto di Ricovero e Cura a Carattere Scientifico (IRCCS), Rome, Italy
}

Primary dystonia is a poorly understood but common movement disorder. Recently, several new primary dystonia genes were identified that provide new insight into dystonia pathogenesis. The GNAL dystonia gene is central for striatal responses to dopamine (DA) and is a component of a molecular pathway already implicated in DOPA-responsive dystonia (DRD). Furthermore, this pathway is also dysfunctional and pathogenically linked to mTOR signaling in L-DOPA-induced dyskinesias (LID). These new data suggest that striatal DA responses are central to primary dystonia, even when symptoms do not benefit from DA therapies. Here we integrate these new findings with current understanding of striatal microcircuitry and other dystonia-causing insults to develop new ideas on the pathophysiology of this incapacitating movement disorder.

\section{Dystonia genetics return to DA}

Movement is central to human behavior, and the neurological diseases that affect motor function are severely incapacitating and a major clinical problem. Primary dystonia (see Glossary) is the third most common neurological movement disorder. It is characterized by involuntary muscle contractions that force the body into abnormal twisted positions and postures or cause patterned or stereotyped movements. Primary dystonia occurs from a normally appearing CNS and lacks an identifiable structural or biochemical cause. This disease has higher frequency amongst individuals who undertake intensive, long-term motor repetitions to enhance their fine motor skills, such as musicians [1]. This relationship associates dystonia with plasticity or over-training of the motor circuits. In addition, the lack of a gross pathological correlate also supports the theory that primary dystonia is a functional disease of abnormal neurochemistry, wiring, or physiology. However, there is no consistent neuropharmacological modification

Corresponding author: Pisani, A. (pisani@uniroma2.it).

Keywords: dystonia; striatum; dopamine; signal transduction; GNAL/G $\alpha$ (olf); mTOR.

0166-2236/\$ - see front matter

(C) 2013 Elsevier Ltd. All rights reserved. http://dx.doi.org/10.1016/j.tins.2013.09.003 of primary dystonia symptoms and the underlying neurobiological defects are largely unknown [2-4].

It is also clear that genetics influence the development of dystonia. Several monogenic familial dystonias have been identified, and these provide an unbiased route into understanding the mechanisms of a disease where classical pharmacological and pathological studies largely fail to provide insight. The process from gene identification to mechanistic understanding of dystonia has nevertheless been slow, in particular because the first primary dystonia genes are widely expressed and of unknown function. However, the advent of next-generation sequencing has increased the speed of dystonia gene discovery. Interestingly, two newly identified primary dystonia genes encode proteins with relatively specific expression in the striatal neurons that respond to DA signaling. One of these, GNAL [guanine nucleotide binding protein ( $\mathrm{G}$ protein), alpha activating activity polypeptide, olfactory type], encodes a postsynaptic element of the same signaling cascade already implicated in DRD, despite DRD being classically regarded as mechanistically distinct from primary dystonia. Furthermore, there is also overlap between this dystonia-associated genetic pathway and the molecular cascade associated with LID and neuroleptic induced dyskinesias/dystonias. Considered together, the new genetic information on primary dystonia and molecular mechanisms of hyperkinetic movement disorders suggests that overlapping and converging mechanisms may underlie distinct types of functional dystonias and other hyperkinetic movement disorders.

\footnotetext{
Glossary

DOPA-responsive dystonia (DRD): a form of dystonia caused by DA deficiency that is effectively treated by L-DOPA administration.

Dystonia: the symptom of abnormal involuntary twisting movements or sustained postures caused by coactivation of agonist and antagonist muscle groups.

GNAL/G $\boldsymbol{\alpha}$ (olf): selectively expressed paralog of widely expressed $G N A S / G \alpha s$ stimulatory G protein $\alpha$ subunit. Couples excitatory G-protein-coupled receptors (GPCR) to adenylate cyclase (AC) activation.

L-DOPA (L-3,4-dihydroxyphenylalanine): metabolic precursor to dopamine (DA) that is used therapeutically to elevate dopamine levels for bradykinesia in Parkinson's disease (PD) or DOPA-responsive dystonia.

L-DOPA-induced dyskinesias (LID): side effect of L-DOPA treatment in PD where L-DOPA fails to stably control motor symptoms and instead induces motor fluctuations, most commonly hyperkinetic dyskinesias.

Primary dystonia: neurological motor disease where dystonia occurs without a structural, degenerative, or metabolic explanation.
} 


\section{Characteristics of primary dystonia}

The symptom of abnormal twisting movements, dystonia, occurs within a variety of syndromes. However, many individuals develop dystonia where there is no identifiable cause or etiology to explain the movement disorder. There is no cure for primary dystonia, and most patients are treated by peripheral administration of Botulinum toxin, to prevent muscle hyperactivation, or deep brain stimulation (DBS) that modifies basal ganglia rhythmicity via electrodes implanted into the globus pallidus. However, DBS is variably effective and addressing which locus is the primary defect in primary dystonia has been a contentious issue for decades. At face value, the hyperkinetic nature of dystonia is similar to dyskinesias or choreas associated with striatal dysfunction, but primary dystonia lacks the neurochemical or pathological hallmarks of these conditions. In recent years the idea of primary dystonia as a network disorder has developed, and focus has turned to other brain regions such as the cerebellum and the multilocus hit hypothesis to explain the symptoms [2,5-12].

Primary dystonia is also a heterogeneous condition. Symptoms can develop in children or adults. Symptoms can affect a single body part and only when performing specific movements, or be generalized, affecting multiple body segments and lack task-specificity. Interestingly, symptom variability seems to be a fundamental feature of dystonia rather than a failure to recognize a spectrum of different diseases. Even well-defined monogenic primary dystonias are semi-penetrant and variable in age of onset, focal or generalized symptoms, or may spread from focal to generalized dystonia in the course of disease progression. This consistent variability in primary dystonia is suggestive of a common underlying mechanism, but one that is plastic and sensitive to multiple genetic or environmental modifiers that synergize or cancel out to drive or inhibit symptom development. Here we describe the expected neurobiological consequences of several dystonia-causing genetic mutations and integrate this information with new progress in research into other hyperactive movement disorders. However, a comprehensive review of all dystonia-causing mutations is beyond the scope of this paper and we refer the reader to reviews by Ozelius et al. [2] and Petrucci and Valente [13] for excellent recent descriptions.

\section{Genetic causes of dystonia}

TOR1A dystonia is a $30 \%$ penetrant, autosomal dominant, early-onset primary torsion dystonia. The disease-causing mutation is an in-frame $3 \mathrm{bp}$ deletion in TOR1A (torsin family 1 , member A) and was the first identified primary dystonia mutation [14]. This deletion impairs TOR1A activity but also produces a mutant protein that lacks a single glutamic acid residue that potentially has gain-offunction or dominant negative actions [15]. TOR1A dystonia symptoms often begin in a single limb and tend to generalize throughout the body, although there are instances where the TOR1A mutation produces focal dystonia [16]. The modifiers that underlie incomplete penetrance and symptom variability are unknown, but a complex set of risks and suppressers are hypothesized because there is no simple link between penetrance and a genetic locus or environmental trigger. In 2008, THAP1 (thanatos-associated domain-containing apoptosis-associated protein 1) was discovered as the second primary dystonia gene. THAP1 dystonia is also autosomal dominant primary dystonia with reduced penetrance and mixed symptom severity [17]. The cellular roles of torsinA and THAP1 remain largely unknown, and these are broadly expressed proteins that have not yet led to cellular or neurobiological explanations for primary dystonia.

L-DOPA is a DA replacement therapy that causes dramatic, rapid improvement of DRD. This type of dystonia is classically regarded as a neurometabolic disorder and mechanistically distinct to forms of primary dystonia that otherwise are known to fail to respond to DA drugs [18]. However, similarly to TOR1A and THAP1 dystonias, DRD is early onset, with reduced penetrance, and the symptoms often spread from a single limb to generalized dystonia. Pertinently, DRD also represents a situation where dystonia occurs despite normal brain structure. Most DRD is caused by dominant inheritance of GCH1 (GTP cyclohydrolase 1) mutations $[18,19]$. There are over 100 distinct pathogenic missense, nonsense, deletions, or splicing GCH1 mutations, and it therefore appears that $G C H 1$ haploinsufficiency is sufficient to cause DRD. There are also less frequent recessive forms of DRD caused by mutations in $T H$ (tyrosine hydroxylase) or SPR (sepiapterin reductase). All three DRD genes participate in DA biosynthesis (Figure 1A): $G C H 1$ and $S P R$ act sequentially to synthesize tetrahydrobiopterin $\left(\mathrm{BH}_{4}\right)$, and $\mathrm{BH}_{4}$ is subsequently required for $T H$ to synthesize L-DOPA from tyrosine $[18,20]$. Thus, these genetic data strongly associate depressed striatal DA release with the development of dystonia.

The advent of next-generation sequencing has rapidly increased the rate of dystonia gene discovery. Dominantly inherited mutations that segregate with primary dystonia are now identified in CIZ1 [CDKN1A (Cip1) interacting zinc finger protein 1], ANO3 (anoctamine3), TUBB4 ( $\beta$ tubulin $4 \mathrm{~A}$ ), and GNAL [21-25]. These recent discoveries are especially interesting because both ANO3 and GNAL are enriched in the striatum, the same nucleus affected by the DRD genetic deficiencies.

ANO3 is a member of the anoctamine gene family whose best-characterized members are epithelial chloride channels [26]. Thus, it is possible that ANO3 mutations cause dystonia by directly perturbing intrinsic neuronal physiology, and that the striatum is particularly affected by virtue of higher ANO3 expression. The GNAL protein is $\mathrm{G} \alpha(\mathrm{olf})$, a stimulatory $\alpha$ subunit for heterotrimeric G-protein complexes (guanine nucleotide-binding protein). GNAL/G $\alpha$ (olf) is a paralog of the widely expressed GNAS gene that encodes $\mathrm{G} \alpha(\mathrm{s})$. $\mathrm{G} \alpha(\mathrm{olf})$ or $\mathrm{G} \alpha(\mathrm{s})$ both functionally couple seven transmembrane G-protein-coupled receptors (GPCR) to adenylate cyclase (AC), and this activity is required for GPCR-stimulated cAMP production (Figure 1A). GNAS/ $\mathrm{G} \alpha(\mathrm{s})$ is the predominant stimulatory $\alpha$-subunit in the CNS and is highly expressed throughout cortex, midbrain, cerebellum, and brainstem, whereas $G N A L / G \alpha($ olf $)$ is expressed at lower levels and displays a more selective expression pattern. However, the relative expression of $\mathrm{G} \alpha(\mathrm{s})$ and $\mathrm{G} \alpha(\mathrm{olf})$ is specifically reversed in the striatum, where $\mathrm{G} \alpha(\mathrm{s})$ is virtually absent and $\mathrm{G} \alpha(\mathrm{olf})$ mRNA is higher 
(A)

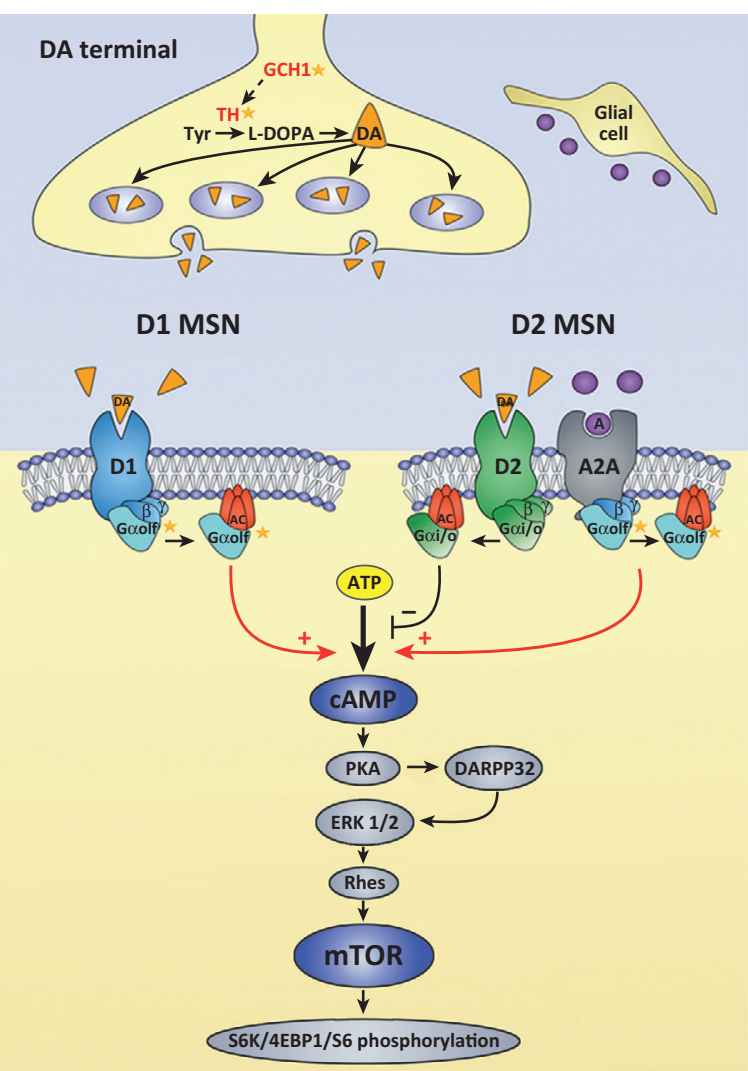

(B)

Gnas/Go(s)

Gnal/Go(olf)
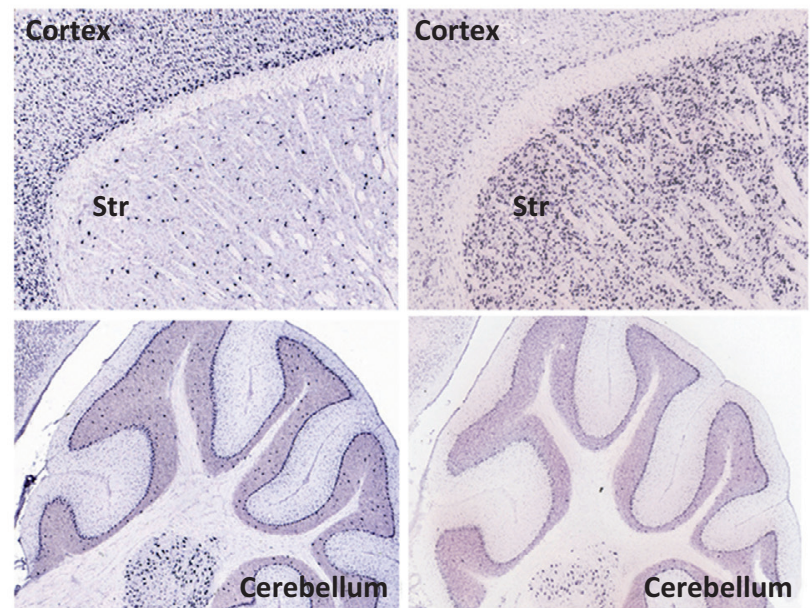

TRENDS in Neurosciences

Figure 1. A dystonia-associated striatal signaling cascade. (A) Dystonia-causing loss-of-function mutations (yellow stars) in GCH1 (GTP cyclohydrolase 1), TH (tyrosine hydroxylase) [18,19], and GNAL/G $\alpha$ (olf) [22] intersect to suppress striatal dopamine (DA) signaling to adenylate cyclase (AC), cAMP, and protein kinase A (PKA). GCH1 and $T H($ red) are presynaptic in the terminals of nigrostriatal projection neurons where they participate in DA biosynthesis. There are two classes of DA receptor, D1DR-like ('D1' - D1DR and D5DR) and D2DR-like ('D2' - D2DR, D3DR, and D4DR) that mediate postsynaptic DA responses. The D1DR and D2DR genes are highly expressed by medium spiny neurons (MSN) of the direct and indirect pathways, respectively, although other DA receptor genes are more broadly expressed. Striatal D1DR-like receptors are excitatory and interact with GNAL/G $\alpha$ (olf) (turquoise) to activate AC. Striatal adenosine 2A receptors (A2A) are specifically expressed by D2DR-bearing MSN, and also couple to $G N A L / G \alpha$ (olf) and cAMP production. The inhibitory class of DA receptors, D2DR-like, instead interact with $\mathrm{G} \alpha(\mathrm{i})$ - or $\mathrm{G} \alpha(\mathrm{o})$-containing $\mathrm{G}$ proteins that inhibit cAMP production, among other targets. Therefore, D1DR-like and A2AR activation have the opposite action to D2DR-like, although the receptors do not necessarily directly compete and there is evidence for extra complexity due to receptor dimerization $[39,40]$. The downstream targets of striatal PKA activation are varied, but many PKAmediated effects require phosphorylation of the DARPP-32 protein and post-translational modification of receptors, ion channels, and altered gene transcription [67]. The activation of extracellular signal-regulated kinases 1/2 (ERK1/2) is also a well-characterized response to PKA activation in MSN. Furthermore, L-DOPA treatment in the DAdepleted condition of Parkinson's disease (PD) provides an example where this dystonia-associated pathway pathologically activates mTOR and associated protein translation machinery via DARPP-32, ERK1/2, and the striatum-specific Rhes G protein [61,62,64]. Additional abbreviations: 4EBP1, eukaryotic translation initiation factor 4Ebinding protein 2 (EIF4EBP1); S6, ribosomal protein S6; S6K, S6 kinase. (B) Gnal encodes the stimulatory heterotrimeric G protein $\alpha$-subunit expressed by most striatal neurons. Images of Gnas and Gnal expression in the mouse cortex, striatum, and cerebellum are from the Allen Mouse Brain Atlas [68]. In situ hybridization signals for Gnas mRNA (left panels: http://mouse brain-map.org/experiment/show/510) are seen in the majority of cortical and cerebellar neurons, but are present in only a small subset of striatal neurons that are probably cholinergic interneurons [29]. By contrast, Gnal in situ hybridization signals (right panels: http://mouse.brain-map.org/experiment/show/ 69734482) are clearly detected in the majority of striatal neurons and are stronger than in other brain areas.

than in other CNS areas [27-29] (Figure 1B). Thus, striatal neurons are specifically dependent on $G N A L / \mathrm{G} \alpha$ (olf) for GPCR stimulation of cAMP levels because they lack GNAS/G $\alpha(\mathrm{s})[27,30]$. Furthermore, functional analyses suggest that dystonia-causing mutations suppress $\mathrm{G} \alpha(\mathrm{olf})$ activity [22] and the association between GNAL mutations and dystonia has been confirmed by independent laboratories and in different ethnic backgrounds [23]. Thus, these data provide strong evidence that $G N A L$-associated primary dystonia develops because of impaired striatal signal transduction.

\section{The striatum is the input station for basal ganglia movement processing}

A consideration of striatal neuron connectivity and pharmacology is needed to translate how $G C H 1, T H$, or $G N A L$ mutations affect CNS function and cause dystonia. The striatum filters a massive cortical and thalamic excitatory glutamatergic signal into an inhibitory GABAergic output that ultimately determines movement/action (Figure 2). The striatum is also the site where DA release promotes movement by signaling to striatal neurons. The majority of striatal neurons are medium spiny neurons (MSN) that project to basal ganglia output nuclei. There are two categories of MSN: the 'direct pathway' MSN that project to the substantia nigra pars reticulata/internal globus pallidus (entopeduncular nucleus of rodents), and the MSN forming the 'indirect pathway' that project to the external globus pallidus (globus pallidus of rodents) [31,32]. Pioneering studies determined that direct pathway MSN express high levels of the D1 DA receptor gene $(D 1 D R)$, whereas expression of both D2DR and adenosine 2 receptors $(\mathrm{A} 2 \mathrm{AR})$ is the hallmark of MSN of the indirect pathway [33,34]. The striatum also contains several 


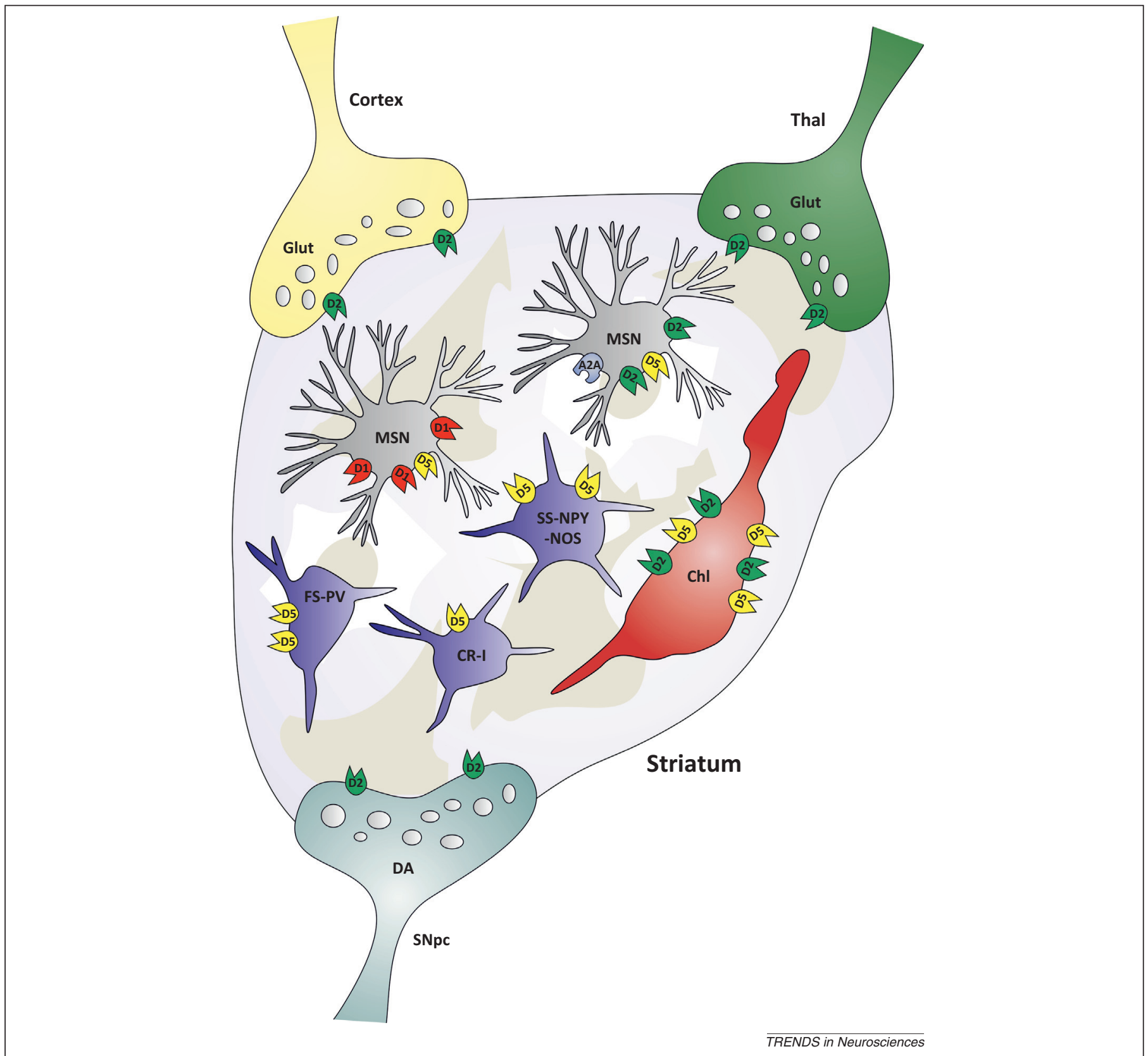

Figure 2. Microcircuitry of the striatum. The striatum is the input nucleus of the basal ganglia motor circuit and integrates glutamatergic (Glut) inputs from the cortex and thalamus (Thal). The striatum contains at least six distinct classes of neuron. The majority are GABAergic medium spiny neurons (MSN) that send axons to basal ganglia

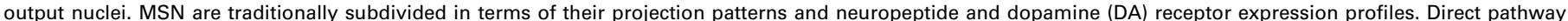

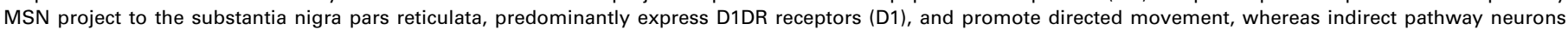
project to the globus pallidus, predominantly express receptors D2DR (D2) and A2AR (adenosine 2 receptor; A2A), and their activity appears to suppress the drive to movement [69]. The striatum also contains several classes of interneuron. There are at least three distinct types of GABAergic interneurons: fast-spiking (FS) parvalbumin

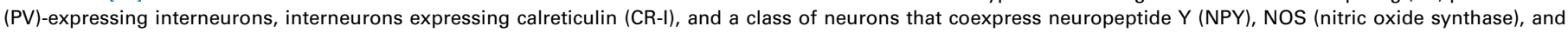
somatostatin (SS). There are also giant interneurons that release acetylcholine (Chl). DA is released in the striatum from axon terminals that originate in the substantia nigra pars compacta $(\mathrm{SNpc})$. DA is a centrally-important modulator of striatal neuron activity via a complex series of events mediated by receptors present on the presynaptic terminals of striatal inputs, on striatal projection neurons and on striatal interneurons. Notably, although excitatory D5DRs (D5, yellow) are expressed at low levels, they are present on many striatal neurons and are higher-affinity DA receptors [70], thus further complicating simple predictions for how DA modifies the activity of different striatal neuron subtypes [36].

classes of GABAergic interneurons that express DA receptors, as well as DA-sensitive cholinergic interneurons that output rhythmic acetylcholine release and also modulate DA levels. Thus, a complex mix of glutamate, adenosine, DA, acetylcholine, GABA, and several neuropeptides, modify striatal control of movement $[35,36]$ (Figure 2). The complexity of this neuronal circuitry is a major challenge for a unifying theory of striatal function and neurotransmitter actions. However, it is clear that striatal DA promotes movement, as classically evidenced by the bradykinesia produced when DA neurons degenerate in Parkinson's disease (PD), whereas degeneration of striatal neurons, with a preference for indirect MSN loss, drives the hyperkinetic motor disturbance of Huntington's disease.

DA receptors are subdivided into two groups: 'excitatory' D1DR-like, comprising the D1DR and D5DR proteins, and 'inhibitory' D2DR-like including the products of the 
$D 2 D R, D 3 D R$, and $D 4 D R$ genes and their splice variants. The different classes of DA receptor couple to different $G$ proteins, and thus DA has different effects depending on which receptors are present (Figure 1A). In a traditional model of basal ganglia function, the direct pathway MSN facilitates movement whereas activation of indirect pathway MSN represses movement. This model also postulates that DA differentially modulates these pathways by stimulating, via the excitatory D1DR, direct pathway MSN and suppressing the indirect pathway MSN (via the D2DR). However, the picture is more complex than represented by this model. For example, there is presynaptic expression of D2DR on DA and glutamatergic terminals. Furthermore, the high-affinity D5DR is expressed by both types of MSN and striatal interneurons [35,36], and there is evidence for coexpression of $D 1 D R$ and $D 2 D R$ genes in a subset of MSN projection neurons [37-39] (Figures 1A,2). There are also other neurotransmitter receptors that signal into DA-sensitive signal transduction cascades, most notably the striatal A2AR that couple to $G N A L / G \alpha$ (olf) and cAMP production, and can heterodimerize with D2DR [40] (Figure 1A). In addition, alongside the model of direct and indirect pathway competition, there is emerging evidence for coordinated activation of both MSN pathways during movement [41,42].

\section{Suppression of a striatal GCH1, TH, and GNAL molecular pathway leads to dystonia}

The GCH1,TH, and GNAL dystonia genes are components of a striatal DA signaling pathway (Figure 1A). GCH1 and $T H$ gene products participate in DA synthesis in the presynaptic portion of this pathway. This biochemical role and the conclusion that impaired DA synthesis causes dystonia derives from the fact that DRD-causing mutations are typically loss-of-function type mutations, L-DOPA administration effectively remediates dystonia symptoms, and there is a demonstrated DA deficiency in the striatum of DRD patients [18,43]. GNAL/G $\alpha$ (olf) functions immediately downstream of striatal D1DR, D5DR, and A2AR, and couples these GPCR subtypes to AC, cAMP, and protein kinase A (PKA) activation [30] (Figure 1A). There are also data from Gnal mice that $\mathrm{G} \alpha$ (olf) expression is rate-limiting for DA stimulation of cAMP [44]. Thus, based on the selective expression, biochemical functions, and the nature of $G C H 1, T H$, and GNAL mutations, it appears that these three genetic causes of dystonia intersect to suppress the D1DR-like/ cAMP/PKA pathway in striatal neurons. It is also interesting to consider that individuals with $G C H 1$ and $T H$ mutations respond well to L-DOPA therapy because this reverses the fundamental problem of DA deficiency, although L-DOPA is unlikely to be as effective when the pathway-suppressing mutation lies downstream of DA or receptors. Together, these data strongly lead to the reemergence of 'old ideas' on the role of the striatum as a key player in dystonia pathophysiology.

\section{Evidence for striatal dysfunction in other forms of dystonia}

Numerous lines of evidence link dopaminergic dysfunction and striatal signaling to the symptom of dystonia $[45,46]$.

\section{Box 1. DA receptor-blocking drugs and dystonia}

Hyperkinetic movement disorders, including dystonia, can appear as a side effect of different psychoactive drugs, including commonly prescribed compounds such as haloperidol. These side effects are most strongly associated with first-generation neuroleptics that competitively block D2DRs. Most D2DR antagonist drug-induced dystonia occurs relatively early in the course of treatment and is reversible on drug discontinuation. However, abnormal involuntary movements also appear after chronic D2DR antagonist use, particularly in the elderly. These motor dysfunctions are often irreversible and difficult to treat, suggesting that long-term D2DR blockade induces permanent changes in striatal physiology. The affinity of the antagonists for D2DR is considered relevant for the drug to bear neuroleptic potential. Of interest, positron emission tomography (PET) studies have shown that optimal antipsychotic response requires approximately 60\% D2DR occupancy; higher occupancy rates are also associated with increased incidence of motor side effects [71-73]. Nevertheless, the appearance of dystonia is variable and only some individuals develop side effects from D2DR blockade. Thus, drug-induced dystonia is another situation where a dystonia-causing insult is well defined, but other modifiers appear to affect the 'penetrance' of dystonia development.

Overall, there is little mechanistic understanding of how D2DR antagonists cause hyperkinetic movements. There is no strong or single-locus genetic explanation for the variability in the occurrence of drug-induced dystonia, although associations are found with polymorphisms in the D2DR gene and in genes encoding the GRIN1 and $2 A$ subunits of the NMDA receptor and the D1DR (reviewed by Teo et al. [74]). Furthermore, the precise involvement of the different D2DR-like receptor subtypes, encoded by the D2DR, D3DR and D4DR genes, remains uncertain, and other neurotransmitters and NMDA and $5-\mathrm{HT}$ receptors emerge as possible alternatives [74]. It seems likely that different mechanisms account for reversible versus irreversible forms of drug-induced dystonia that result from acute and chronic drug administration, respectively. For irreversible druginduced dystonia it is also necessary to consider how long-term use of D2DR antagonists permanently alters striatal physiology and neurotransmission because there is no obvious sign of structural defects in these patients. The idea that long-term neuroleptic use induces maladaptive synaptic plasticity has been proposed [74] but, in general, we are some distance from understanding the molecular details of how pharmacological repression of DA signaling permanently affects the striatum and motor circuitry.

However, the relationship for primary dystonia remains contentious because, by definition, primary dystonia is rarely affected by dopaminergic therapies. The identification of $G N A L / G \alpha$ (olf) as a primary dystonia gene now highlights the idea that dystonia can occur because of defects in striatal signal transduction pathways downstream of DA receptors. There is also evidence from other forms of dystonia to support this theory for defective striatal signaling in dystonia. First, it is well recognized that short-term and long-term pharmacological blockade of D2DR produces dystonia. Although the mechanisms underlying this relatively common side effect remain unclear (Box 1), this further associates dystonia with DA and striatal dysfunction. Furthermore, striatal D2DR availability is decreased in THAP1 dystonia and TOR1A dystonia patients, which are two forms of primary dystonia that rarely respond to DA drugs $[47,48]$. The deficiency in D2DR is also seen in non-manifesting carriers of the TOR $1 A$ mutation, which suggests that these DA receptor deficits are a primary consequence of the dystonia-causing mutation rather than secondary compensation against dystonia. More evidence for defective DA signaling comes from several genetic rodent models of TOR1A dystonia. Mice 
carrying Tor1a mutations have aberrant D2DR-mediated responses that are paralleled by reduced D2DR protein levels and impaired activation of the cognate $G$ proteins [49]. There is also a significant impairment of striatal neuron plasticity to cortical inputs in transgenic mice and rats overexpressing mutant torsinA [50,51], as well as in mice where the dystonia-causing deletion is introduced into the mouse Tor1a gene (DYT1 $\Delta$ gag knock-in mice) [52]. Plasticity defects in DYT1 models are primarily caused by altered D2DR-mediated control of cholinergic neuron function, and in one model were restored by antagonism of A2AR [49,51].

The mechanism(s) that link TOR1A and THAP1 mutations with postsynaptic striatal neurobiological defects are largely unknown. However, evidence from mouse and Drosophila suggests that torsin A is important for trafficking postsynaptic mRNAs from the nucleus to the cytosol [53,54]. Furthermore, there is a genetic interaction reported between Drosophila torsin and DA synthesis [55]. In the case of THAP1 dystonia, the THAP1 protein is a binding partner for Par4, which in turn is an interaction partner of the D2DR $[56,57]$. Thus, both TOR1A and THAP1 dystonia are associated with defective postsynaptic striatal responses to DA, although the evidence points to the D2DR pathway also implicated in drug-induced dystonias (Box 1) rather than the D1DR-like pathway at the intersection of $T H, G C H 1$, and GNAL gene mutations (Figure 1).

\section{Depressed striatal DA signaling leads to dystonia?}

There is a paradox if we consider that suppressed striatal DA signaling causes dystonia, whereas loss of striatal DA in PD instead decreases the drive for voluntary movements and results in bradykinesia. The need to account for additional complexity is also highlighted by the strong relationship between dystonia development and D2DR blockade (Box 1), whereas GCH1, TH, and GNAL mutations intersect to affect the D1DR-like pathway. There are no clear explanations for these paradoxes, although the dual nature of dystonia-causing insults is more consistent with basal ganglia models that focus on coordinated MSN activation [42], rather than competition between direct and indirect pathways, given that this predicts that the dystonia-causing insults should oppositely affect movement. Furthermore, compensatory interactions within the striatal microcircuitry or crosstalk between signal transduction cascades, via mechanisms such as dimerization between A2AR, D1DR, or D2DR proteins [40,58], are one possibility. One other factor to consider is that familial dystonia patients are haploinsufficient rather than null for DA signaling components, and this may induce different compensation than stronger, broader blockade from progressive dopaminergic degeneration in PD. In addition, a developmental component seems likely. Patients with familial dystonia have congenitally blunted DA signaling during childhood acquisition of motor skills when striatal responses may be more plastic than in the case of an adult who develops PD. This theory of age-associated differences in how DA loss affects the striatum and movement is also supported by the more frequent appearance of dystonia in early-onset compared with late-onset forms of PD [59].

\section{Downstream effects of DA signaling; what is learnt from L-DOPA dyskinesia}

This evidence for a complex interplay of compensations in the striatum also highlights that there is more to consider than the top end of striatal signaling to cAMP and PKA (Figure 1A). Furthermore, the downstream elements of DA signaling are implicated in LID, another pathological condition with abnormal hyperkinetic movements. L-DOPA is a DA replacement therapy that compensates for DA loss in $\mathrm{PD}$ and is an important symptomatic treatment for PD patients. However, the clinical response to L-DOPA decreases with disease progression, and patients progressively require increasing L-DOPA doses to maintain therapeutic benefit. Ultimately, L-DOPA fails to stably control motor symptoms and patients exhibit severe fluctuations in motor activity. LIDs are the most common manifestations of motor fluctuations, although dystonia can also become a major complication [60].

Many neurochemical defects exist in the sensitized striatum of patients and animal models with LID. However, hypersensitivity of the D1DR pathway appears to play a central role, and L-DOPA treatment in LID models causes dramatic hyperactivation of D1DR pathway components alongside abnormal movements [60]. There are normal levels of D1DR in LID models, but D1DR activation induces hyperphosphorylation of DARPP-32 (dopamine cAMP regulated phosphoprotein $32 \mathrm{kDa}$; Figure 1A) and other PKA targets. DARPP-32 seems to be an important player in LID and is a phosphatase inhibitor that augments activation of the ERK1 and ERK2 members of the MAPK family (mitogen-activated protein kinases) that alter gene transcription [61]. mTORC1 signaling (Box 2)

\section{Box 2. mTOR signaling and plasticity}

Mechanistic target of rapamycin (mTOR; previously mammalian TOR) was initially identified as the target of the rapamycin toxin that inhibits protein synthesis. mTOR is a ubiquitous and a centrally important regulator of protein synthesis and cell growth. The pathway is sensitive to many external and internal cues, and is regarded as a master integrator of signal transduction cascades. Signals such as the phosphoinositol-3-kinase (PI3K)/Akt pathway or extracellular signal-regulated kinase (ERK) typically converge on the upstream tuberous sclerosis complex proteins (TSC) that act as a GAP (GTPase activating protein) to inactivate the atypical G protein, Rheb, and activate mTOR. mTOR itself is an atypical serine/ threonine kinase, and the activated mTOR kinase operates within mTORC1 or mTORC2 complexes to phosphorylate effectors of mTOR signaling. The best-characterized downstream effect of mTOR activation is $\mathrm{mTORC} 1$ phosphorylation of the p70 ribosomal protein S6 kinase, which in turn activates S6 and elF4E-binding protein 1 (EIF4EBP) to upregulate translation initiation factor activity and protein synthesis [75].

mTOR in the brain is central to how neurons produce long-term durable changes in synaptic strength that depend on new protein synthesis $[65,75,76]$. mTORC1 pathway components are detected at postsynaptic terminals; mTORC1 inhibition by rapamycin inhibits long-term synaptic plasticity in response to electrical or chemical stimulation and impairs long-term memory formation in animals. Conversely, mTORC1 stimulation is associated with dendritic spine formation and synaptogenesis [77]. However, mTORC1 signaling is not only associated with beneficial physiology. TSC1/2 mutations that elevate mTOR pathway activity cause autism and intellectual disability in tuberous sclerosis and, in general, excess mTOR signaling is associated with pathological aberrant and hyperactive circuit connectivity [78]. 


\section{Box 3. Outstanding questions}

- What are the molecular and physiological consequences of mTOR activation in striatal MSN?

- Is hyperactive mTOR also a feature of D1DR pathway-associated dystonia or other dystonias?

- How does suppression or fluctuation of DA signaling in LID/PD hyperactivate the D1DR pathway and cause pathological coupling to $\mathrm{mTOR}$ signaling?

- Is there a relationship between how dystonia is caused by pharmacological D2DR blockade or genetic loss of D1DR-like pathway components?

is also strongly associated with DARRP-32 hyperactivation and with how long-term depletion or fluctuations in striatal DA induce pathogenic reprogramming of a DA signal transduction cascade and LID. This evidence for mTOR involvement in LID derives from the observation that LDOPA treatment strongly induces mTOR activation in $D 1 d r$ gene-expressing MSN of the DA-depleted rodent striatum. By contrast, L-DOPA does not induce mTOR activation in the normal striatal environment, and thus it appears that DA receptor coupling to striatal mTOR activation is a specific response that occurs with suppression of DA signaling [62]. Furthermore, the mTORC1 inhibitor, rapamycin, suppresses abnormal mTORC1 activation and dyskinesia-type behaviors in mouse and rat models $[62,63]$. This pathogenic coupling of D1DR signal transduction to mTORC1 occurs via DARPP-32 [61], hyperactivation of ERK1, ERK2, and Rhes, a striatalspecific mTOR activating protein that is homologous to the ubiquitously expressed Rheb [64] (Figure 1A, Box 2). Thus, research on models of LID has determined that DA loss can remodel D1DR-like signaling to inappropriately activate mTOR, which is an effector regarded as being centrally important for long-term changes in synaptic plasticity (Box 2). These data provide an intriguing hypothesis for how $T H$ mutations and GCH1 or GNAL haploinsufficiency might affect striatal plasticity and cause dystonia. Outstanding questions are listed in Box 3.

\section{Concluding remarks}

The identification of dystonia-causing genetic mutations is continuing to lead the way in understanding the molecular and neurobiological drivers of primary dystonia. There is an emerging theme where multiple dystonia-causing mutations converge to affect striatal signaling and signal transduction pathways similarly. They also suggest a previously unrecognized relationship between DRD and primary dystonia. This is an important insight because it suggests that a focus on how congenital DA deficiency affects striatal development and plasticity may have general importance for understanding primary dystonia. It will also be fascinating to determine whether primary dystonia is associated with pathogenic activation of mTOR signaling that is already implicated in LID. The mTORC1 molecular mechanism is a 'shoe' that fits the predictions and expectations for the mechanisms underlying dystonia: it is modulated in the striatum by DA pathways and downstream of verified dystonia genes, it is a master regulator that integrates cellular homeostasis with environment cues, it is an important mediator of synaptic plasticity, and mTOR hyperactivation is a known pathogenic event in the development of functional CNS disease $[65,66]$. Pharmacological manipulation of mTOR is currently under trial for genetically defined autism spectrum disorders. Potentially, therapies designed for these conditions could be repositioned for LID and may be appropriate for other hyperkinetic movement disorders. Furthermore, Rhes and $G \alpha$ (olf) are relatively specific striatal signal transduction proteins implicated in GCH1/TH/GNAL dystonia and LIDs. The striatal specific expression raises the possibility that it may be possible to therapeutically target striatal dysfunction without generally disturbing CNS signal transduction. Furthermore, the discovery of specialized striatal signal transduction molecules that appear to couple to synaptic plasticity can reconcile how dystonia can specifically affect movement while sparing cognitive and affective circuits.

\section{Acknowledgments}

We would like to acknowledge COST (European Cooperation in Science and Technology) Action BM1101 for facilitating discussion and promoting networking. The authors also acknowledge financial support from the Foundation for Dystonia Research (FDR) to R.G., K.G., A.P., the Dystonia Medical Research Foundation (DMRF) to A.P. and previous support of K.G. and R.G., and the Italian Ministry of Health (Progetto Finalizzato) to A.P. K.G. received funding from the Deutsche Forschungsgemeinschaft, the IZKF programme of the University of Tuebingen and the Elitepostdoc programme Baden-Württemberg.

\section{References}

1 Altenmuller, E. and Jabusch, H.C. (2010) Focal dystonia in musicians: phenomenology, pathophysiology and triggering factors. Eur. J. Neurol. 17 (Suppl. 1), 31-36

2 Ozelius, L.J. et al. (2011) Milestones in dystonia. Mov. Disord. 26, 1106-1126

3 Tanabe, L.M. et al. (2009) Primary dystonia: molecules and mechanisms. Nat. Rev. Neurol. 5, 598-609

4 Breakefield, X.O. et al. (2008) The pathophysiological basis of dystonias. Nat. Rev. Neurosci. 9, 222-234

5 Ramdhani, R.A. and Simonyan, K. (2013) Primary dystonia: conceptualizing the disorder through a structural brain imaging lens. Tremor Other Hyperkinet. Mov. (N.Y.) 3, tre-03-152-3638-4

6 Filip, P. et al. (2013) Dystonia and the cerebellum: a new field of interest in movement disorders? Clin. Neurophysiol. 124, 1269-1276

7 Beck, S. and Hallett, M. (2011) Surround inhibition in the motor system. Exp. Brain Res. 210, 165-172

8 Niethammer, M. et al. (2011) Hereditary dystonia as a neurodevelopmental circuit disorder: evidence from neuroimaging. Neurobiol. Dis. 42, 202-209

9 Stamelou, M. et al. (2012) The non-motor syndrome of primary dystonia: clinical and pathophysiological implications. Brain 135, 1668-1681

10 Hallett, M. (2006) Pathophysiology of writer's cramp. Hum. Mov. Sci. $25,454-463$

11 Quartarone, A. et al. (2006) Task-specific hand dystonia: can too much plasticity be bad for you? Trends Neurosci. 29, 192-199

12 Blake, D.T. et al. (2002) Sensory representation abnormalities that parallel focal hand dystonia in a primate model. Somatosens. Mot. Res. 19, 347-357

13 Petrucci, S. and Valente, E.M. (2013) Genetic issues in the diagnosis of dystonias. Front. Neurol. 4, 34

14 Ozelius, L.J. et al. (1997) The early-onset torsion dystonia gene (DYT1) encodes an ATP-binding protein. Nat. Genet. 17, 40-48

15 Goodchild, R.E. et al. (2005) Loss of the dystonia-associated protein TorsinA selectively disrupts the neuronal nuclear envelope. Neuron 48, 923-932

16 Opal, P. et al. (2002) Intrafamilial phenotypic variability of the DYT1 dystonia: from asymptomatic TOR1A gene carrier status to dystonic storm. Mov. Disord. 17, 339-345 
17 Fuchs, T. et al. (2009) Mutations in the THAP1 gene are responsible for DYT6 primary torsion dystonia. Nat. Genet. 41, 286-288

18 Asmus, F. and Gasser, T. (2010) Dystonia-plus syndromes. Eur. J. Neurol. 17 (Suppl. 1), 37-45

19 Ichinose, H. et al. (1994) Hereditary progressive dystonia with marked diurnal fluctuation caused by mutations in the GTP cyclohydrolase I gene. Nat. Genet. 8, 236-242

20 Clot, F. et al. (2009) Exhaustive analysis of BH4 and dopamine biosynthesis genes in patients with Dopa-responsive dystonia. Brain $132,1753-1763$

21 Charlesworth, G. et al. (2012) Mutations in ANO3 cause dominant craniocervical dystonia: ion channel implicated in pathogenesis. Am. J. Hum. Genet. 91, 1041-1050

22 Fuchs, T. et al. (2012) Mutations in GNAL cause primary torsion dystonia. Nat. Genet. 45, 88-92

23 Vemula, S.R. et al. (2013) Role of Galpha(olf) in familial and sporadic adult-onset primary dystonia. Hum. Mol. Genet. 22, 2510-2519

24 Lohmann, K. et al. (2012) Whispering dysphonia (DYT4 dystonia) is caused by a mutation in the TUBB4 gene. Ann. Neurol. http:// dx.doi.org/10.1002/ana.23829

25 Hersheson, J. et al. (2012) Mutations in the autoregulatory domain of beta-tubulin 4a cause hereditary dystonia. Ann. Neurol. http:// dx.doi.org/10.1002/ana.23832

26 Hartzell, H.C. et al. (2009) Anoctamin/TMEM16 family members are $\mathrm{Ca}^{2+}$-activated $\mathrm{Cl}^{-}$channels. J. Physiol. 587, 2127-2139

27 Zhuang, X. et al. (2000) G(olf)alpha mediates dopamine D1 receptor signaling. J. Neurosci. 20, RC91

28 Kull, B. et al. (2000) Adenosine A(2A) receptors are colocalized with and activate g(olf) in rat striatum. Mol. Pharmacol. 58, 771-777

29 Herve, D. et al. (2001) Galpha(olf) levels are regulated by receptor usage and control dopamine and adenosine action in the striatum. $J$ Neurosci. 21, 4390-4399

30 Corvol, J.C. et al. (2001) Galpha(olf) is necessary for coupling D1 and A2a receptors to adenylyl cyclase in the striatum. J. Neurochem. 76, $1585-1588$

31 Gerfen, C.R. (1984) The neostriatal mosaic: compartmentalization of corticostriatal input and striatonigral output systems. Nature 311 461-464

32 Graybiel, A.M. (1990) Neurotransmitters and neuromodulators in the basal ganglia. Trends Neurosci. 13, 244-254

33 Gerfen, C.R. et al. (1990) D1 and D2 dopamine receptor-regulated gene expression of striatonigral and striatopallidal neurons. Science 250, 1429-1432

34 Schiffmann, S.N. et al. (2007) Adenosine A2A receptors and basal ganglia physiology. Prog. Neurobiol. 83, 277-292

35 Surmeier, D.J. and Graybiel, A.M. (2012) A feud that wasn't: acetylcholine evokes dopamine release in the striatum. Neuron 75 , $1-3$

36 Tritsch, N.X. and Sabatini, B.L. (2012) Dopaminergic modulation of synaptic transmission in cortex and striatum. Neuron 76, 33-50

37 Rivera, A. et al. (2002) Molecular phenotype of rat striatal neurons expressing the dopamine D5 receptor subtype. Eur. J. Neurosci. 16, 2049-2058

38 Rashid, A.J. et al. (2007) D1-D2 dopamine receptor heterooligomers with unique pharmacology are coupled to rapid activation of $\mathrm{Gq} / 11$ in the striatum. Proc. Natl. Acad. Sci. U.S.A. 104, 654-659

39 Perreault, M.L. et al. (2011) The dopamine D1-D2 receptor heteromer in striatal medium spiny neurons: evidence for a third distinct neuronal pathway in basal ganglia. Front. Neuroanat. 5, 31

40 Ferré, S. et al. (2004) Adenosine A2A-dopamine D2 receptor-receptor heteromers. Targets for neuro-psychiatric disorders. Parkinsonism Relat. Disord. 10, 265-271

41 Bagetta, V. et al. (2011) Dopamine-dependent long-term depression is expressed in striatal spiny neurons of both direct and indirect pathways: implications for Parkinson's disease. J. Neurosci. 31, 12513-12522

42 Cui, G. et al. (2013) Concurrent activation of striatal direct and indirect pathways during action initiation. Nature 494, 238-242

43 Furukawa, Y. et al. (1999) Striatal biopterin and tyrosine hydroxylase protein reduction in dopa-responsive dystonia. Neurology 53, 1032-1041

44 Corvol, J.C. et al. (2007) Quantitative changes in Galphaolf protein levels, but not D1 receptor, alter specifically acute responses to psychostimulants. Neuropsychopharmacology 32, 1109-1121
45 Perlmutter, J.S. and Mink, J.W. (2004) Dysfunction of dopaminergic pathways in dystonia. Adv. Neurol. 94, 163-170

46 Wichmann, T. (2008) Commentary: dopaminergic dysfunction in DYT1 dystonia. Exp. Neurol. 212, 242-246

47 Carbon, M. et al. (2009) Abnormal striatal and thalamic dopamine neurotransmission: genotype-related features of dystonia. Neurology $72,2097-2103$

48 Asanuma, K. et al. (2005) Decreased striatal D2 receptor binding in non-manifesting carriers of the DYT1 dystonia mutation. Neurology 64, 347-349

49 Napolitano, F. et al. (2010) Dopamine D2 receptor dysfunction is rescued by adenosine A2A receptor antagonism in a model of DYT1 dystonia. Neurobiol. Dis. 38, 434-445

50 Grundmann, K. et al. (2012) Generation of a novel rodent model for DYT1 dystonia. Neurobiol. Dis. 47, 61-74

51 Martella, G. et al. (2009) Impairment of bidirectional synaptic plasticity in the striatum of a mouse model of DYT1 dystonia: role of endogenous acetylcholine. Brain 132, 2336-2349

52 Dang, M.T. et al. (2012) An anticholinergic reverses motor control and corticostriatal LTD deficits in Dyt1 DeltaGAG knock-in mice. Behav. Brain Res. 226, 465-472

53 Jokhi, V. et al. (2013) Torsin mediates primary envelopment of large ribonucleoprotein granules at the nuclear envelope. Cell Rep. 3, 988995

54 Speese, S.D. et al. (2012) Nuclear envelope budding enables large ribonucleoprotein particle export during synaptic Wnt signaling. Cell 149, 832-846

55 Wakabayashi-Ito, N. et al. (2011) dtorsin, the Drosophila ortholog of the early-onset dystonia TOR1A (DYT1), plays a novel role in dopamine metabolism. PLoS ONE 6, e26183

56 Roussigne, M. et al. (2003) THAP1 is a nuclear proapoptotic factor that links prostate-apoptosis-response-4 (Par-4) to PML nuclear bodies. Oncogene 22, 2432-2442

57 Park, S.K. et al. (2005) Par-4 links dopamine signaling and depression. Cell 122, 275-287

58 Hasbi, A. et al. (2010) Heteromerization of dopamine D2 receptors with dopamine D1 or D5 receptors generates intracellular calcium signaling by different mechanisms. Curr. Opin. Pharmacol. 10, 93-99

59 Wickremaratchi, M.M. et al. (2011) The motor phenotype of Parkinson's disease in relation to age at onset. Mov. Disord. 26, 457-463

60 Santini, E. et al. (2008) Parkinson's disease: levodopa-induced dyskinesia and signal transduction. FEBS J. 275, 1392-1399

61 Santini, E. et al. (2012) Dopamine- and cAMP-regulated phosphoprotein of 32-kDa (DARPP-32)-dependent activation of extracellular signal-regulated kinase (ERK) and mammalian target of rapamycin complex 1 (mTORC1) signaling in experimental parkinsonism. J. Biol. Chem. 287, 27806-27812

62 Santini, E. et al. (2009) Inhibition of mTOR signaling in Parkinson's disease prevents L-DOPA-induced dyskinesia. Sci. Signal. 2, ra36

63 Decressac, M. and Björklund, A. (2013) mTOR inhibition alleviates LDOPA-induced dyskinesia in Parkinsonian rats. J. Parkinsons Dis. 3, $13-17$

64 Subramaniam, S. et al. (2012) Rhes, a striatal-enriched small G protein, mediates mTOR signaling and L-DOPA-induced dyskinesia. Nat. Neurosci. 15, 191-193

65 Hoeffer, C.A. and Klann, E. (2010) mTOR signaling: at the crossroads of plasticity, memory and disease. Trends Neurosci. 33, 67-75

66 Han, J.M. and Sahin, M. (2011) TSC1/TSC2 signaling in the CNS. FEBS Lett. 585, 973-980

67 Yger, M. and Girault, J.A. (2011) DARPP-32, jack of all trades... master of which? Front. Behav. Neurosci. 5, 56

68 Lein, E.S. et al. (2007) Genome-wide atlas of gene expression in the adult mouse brain. Nature 445, 168-176

69 Gerfen, C.R. and Surmeier, D.J. (2011) Modulation of striatal projection systems by dopamine. Annu. Rev. Neurosci. 34, 441-466

70 Sunahara, R.K. et al. (1991) Cloning of the gene for a human dopamine D5 receptor with higher affinity for dopamine than D1. Nature 350, 614-619

71 Farde, L. et al. (1992) Positron emission tomographic analysis of central D1 and D2 dopamine receptor occupancy in patients treated with classical neuroleptics and clozapine. Relation to extrapyramidal side effects. Arch. Gen. Psychiatry 49, 538-544 
72 Kapur, S. et al. (2000) Relationship between dopamine D(2) occupancy, clinical response, and side effects: a double-blind PET study of firstepisode schizophrenia. Am. J. Psychiatry 157, 514-520

73 Nordstrom, A.L. et al. (1993) Central D2-dopamine receptor occupancy in relation to antipsychotic drug effects: a double-blind PET study of schizophrenic patients. Biol. Psychiatry 33, 227-235

74 Teo, J.T. et al. (2012) Tardive dyskinesia is caused by maladaptive synaptic plasticity: a hypothesis. Mov. Disord. 27, 1205-1215

75 Costa-Mattioli, M. et al. (2009) Translational control of long-lasting synaptic plasticity and memory. Neuron $61,10-26$
76 Richter, J.D. and Klann, E. (2009) Making synaptic plasticity and memory last: mechanisms of translational regulation. Genes Dev. 23, $1-11$

$77 \mathrm{Li}$, N. et al. (2010) mTOR-dependent synapse formation underlies the rapid antidepressant effects of NMDA antagonists. Science 329, 959964

78 Santini, E. and Klann, E. (2011) Dysregulated mTORC1-dependent translational control: from brain disorders to psychoactive drugs. Front. Behav. Neurosci. 5, 76 